\title{
Understanding Factors in Group B Streptococcus Late-Onset Disease
}

\author{
Alberto Berardi (iD)' \\ Viola Trevisani ${ }^{2}$ \\ Antonella Di Caprio ${ }^{2}$ \\ Jenny Bua ${ }^{3}$ \\ Mariachiara China ${ }^{4}$ \\ Barbara Perrone ${ }^{5}$ \\ Rossella Pagano ${ }^{6}$ \\ Laura Lucaccioni ${ }^{7}$ \\ Silvia Fanaro ${ }^{8}$ \\ Lorenzo lughetti ${ }^{2,7}$ \\ Licia Lugli' \\ Roberta Creti ${ }^{9}$
}

'Terapia Intensiva Neonatale, Azienda OspedalieroUniversitaria Policlinico, Modena, Italy; ${ }^{2}$ Scuola di Specializzazione in Pediatria, Università di Modena \& Reggio Emilia, Modena, Italy; ${ }^{3}$ Terapia Intensiva Neonatale, IRCCS Azienda Ospedaliero Universitaria "Burlo Garofalo", Trieste, Italy; "Terapia Intensiva Neonatale, Ospedale Infermi, Rimini, Italy; ${ }^{5}$ Terapia Intensiva Neonatale, Azienda Ospedaliero Universitaria Ospedali Riuniti, Ancona, Italy; ${ }^{6}$ Unità Operativa di Pediatria, Civile Sassuolo, Sassuolo, Italy; ${ }^{7}$ Unità

Operativa di Pediatria, Azienda Ospedaliero-Universitaria Policlinico, Modena, Italy; ${ }^{8}$ Terapia Intensiva Neonatale, Azienda Ospedaliero-Universitaria S. Anna, Ferrara, Italy; ${ }^{9}$ Reparto di Antibiotico Resistenza e Patogeni Speciali (AR-PS), Dipartimento di Malattie Infettive, Istituto

Superiore di Sanità, Rome, Italy

\section{Video abstract}

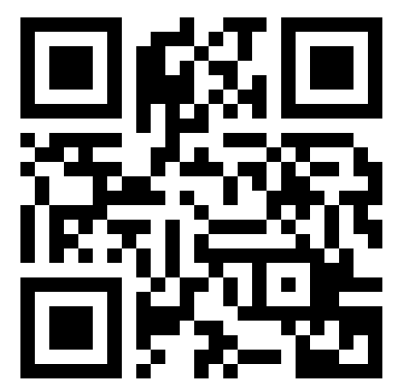

Point your SmartPhone at the code above. If you have a $Q R$ code reader the video abstract will appear. Or use: https://youtu.be/Twhdky4vgV|

Correspondence: Alberto Berardi Tel +390594222522

Fax +3905942223770

Email alberto.berardi@unimore.it

\begin{abstract}
Group B streptococcus (GBS) infection remains a leading cause of sepsis, pneumonia, and meningitis in infants. Rates of GBS early onset disease have declined following the widcespread use of intrapartum antibiotic prophylaxis; hence, late-onset infections (LOGBS) are currently a common presentation of neonatal GBS dicsease. The pathogenesis, mode of transmission, and risk factors associated with LOGBS are unclear, which interfere with effective prevention efforts. GBS may be transmitted from the mother to the infant at the time of delivery or during the postpartum period via contaminated breast milk, or as nosocomial or community-acquired infection. Maternal GBS colonization, prematurity, young maternal age, HIV exposure, and ethnicity (Black) are identified as risk factors for LOGBS disease; however, further studies are necessary to confirm additional risk factors, if any, for the implementation of effective prevention strategies. This narrative review discusses current and previous studies that have reported LOGBS. Few welldesigned studies have described this condition; therefore, reliable assessment of maternal GBS colonization, breastfeeding, and twin delivery as risk factors for LOGBS remains limited.
\end{abstract}

Keywords: neonatal sepsis, prevention, infant, meningitis, $\mathrm{CC} 17$

\section{Introduction}

Group B streptococcus (GBS or Streptococcus agalactiae), a gram-positive pathogen that belongs to the Lancefield group B class of streptococci, is a common gastrointestinal and urogenital commensal in adults, which may occasionally precipitate life-threatening infections in neonates, pregnant women, elderly individuals, or in adults with comorbidities. ${ }^{1,2}$ GBS colonizes the vagina or vaginal/ rectal (VR) sites in 10-30\% of pregnant women and frequently causes urinary tract infections, chorioamnionitis, and postpartum endometritis. GBS is also associated with poor pregnancy outcomes, including spontaneous abortion, stillbirth, and preterm birth. ${ }^{1,3,4}$

Since the 1970s, GBS has emerged as a leading cause of sepsis, pneumonia, and meningitis in infants in the USA. ${ }^{1}$ The worldwide burden of GBS infection remains significant, ${ }^{5}$ and GBS affects approximately 320,000 neonates (90,000 of which die) across low-, middle-, and high-income countries. ${ }^{6}$ GBS infections significantly contribute to the global pediatric mortality rates (children aged $<5$ years), with the highest rates observed in low-income countries. ${ }^{7}$ Studies performed since the $1970 \mathrm{~s}$ have identified maternal VR carriage as a primary risk factor (RF) for neonatal 
colonization and infection, ${ }^{8-14}$ and research has focused on strategies to prevent mother-to-infant transmission. ${ }^{15-17}$

Neonatal GBS infections are categorized into earlyonset disease (EOGBS, presenting at 0-6 days of life) and late-onset disease (LOGBS, presenting at 7-90 days of life). ${ }^{3}$ GBS infections may occur beyond 90 days and within 6 months of life (ultra-late onset disease [ULOGBS]). Cohort studies have shown that LOGBS and ULOGBS are identical clinical and bacteriological entities, except for prematurity, which is more commonly associated with ULOGBS. ${ }^{18}$ We focus on LOGBS; therefore, ULOGBS is not discussed in this review.

Early studies report that the rates of EOGBS disease were approximately 4-fold higher than those of LOGBS. Clinical trials performed since the 1980s report that administration of intravenous ampicillin or penicillin (intrapartum antibiotic prophylaxis [IAP]) to mothers at a high risk of GBS transmission to their newborn could effectively prevent EOGBS. IAP has significantly reduced the rates of EOGBS; ${ }^{3}$ the incidence of EOGBS in the USA was as high as 5 cases per 1000 live births prior to the widespread availability of IAP $^{19}$ and has declined significantly post-IAP, with the trend continuing even in recent years $(0.37 / 1000$ live births in 2006 and $0.23 / 1000$ live births in 2015). The LOGBS rates among infants have stabilized in the USA (mean 0.31/1000 live births) and are now higher than those of EOGBS. ${ }^{20}$ Therefore, investigation of RFs associated with LOGBS is important because in some high income countries LOGBS is now the most common presentation of neonatal GBS disease.

\section{Pathogenesis and Clinical Presentation of Early- and Late-Onset Group B Streptococcal Disease}

EOGBS and LOGBS are similar with regard to bacterial attachment to the epithelial surface, invasion, and bacteremia and differ only with regard to the timing and mode of exposure. EOGBS and LOGBS differ in their clinical presentation, mortality, morbidity, epidemiological characteristics, and percentage of GBS serotypes that cause invasive infections. The route of transmission of GBS in EOGBS is well known, whereas that in LOGBS remains unclear. EOGBS is vertically acquired, and maternal anogenital colonization is a prerequisite in such cases. Neonatal exposure occurs in utero (hours or a few days before birth) or during passage through the birth canal. Before birth, GBS are aspirated into the lungs (exposure of the respiratory epithelial surface) with subsequent invasion of pulmonary vessels. Aspiration and swallowing of vaginal secretions result in bacterial adherence to respiratory and gastrointestinal mucous membranes with subsequent bacterial invasion of the bloodstream, ${ }^{1}$ and infants typically present with pneumonia or sepsis at birth or soon thereafter. ${ }^{1,8}$ In contrast, LOGBS can result from mucosal colonization of GBS at the time of delivery or postpartum, from the mother or from other sources. Early studies indicate that approximately $40 \%$ of neonates with GBS colonization at birth continue to show intestinal colonization even at 12 weeks of life and that intestinal GBS colonization is an important precursor of LOGBS. ${ }^{14,21}$ Studies in animal models have shown that oral administration of GBS leads to systemic disease. ${ }^{22}$ The pathogenesis of LOGBS involves GBS adhesion to mucosal surfaces, followed by invasion of the epithelium and subsequently the bloodstream. Factors that mediate persistent intestinal colonization or promote the shift from intestinal colonization to invasive GBS disease remain unknown. A recent study by Vaz et al in a murine model with exposed offspring and juvenile mice reported the kinetics of gastrointestinal colonization by GBS, progression to invasive disease, and the role of GBS-specific immunoglobulin (Ig)G production. The experimental animals showed prolonged gastrointestinal colonization and developed invasive disease, following both perinatal (21\% of cases) and postnatal exposure to GBS ( $27 \%$ of cases). ${ }^{23}$

Breast milk is considered a possible source of LOGBS and may account for sustained transmission of GBS to the newborn with consequent promotion of gut translocation and LOGBS. ${ }^{24}$ However, whether breast milk is only a marker for high levels of neonatal nasopharyngeal GBS colonization is unclear. ${ }^{2,24-26}$

LOGBS can be also transmitted from non-maternal sources (such as from caregivers); it is shown that all cases of LOGBS are not necessarily associated with maternal colonization. ${ }^{27,28}$ Nosocomial GBS transmission through healthcare workers was more common some decades earlier when post-parturient mothers and infants typically remained in the hospital $\geq 1$ week. ${ }^{11,29,30}$ However, currently full- or late-preterm neonates remain in the hospital only for a few days and "room in" with their mothers; therefore, predominant routes of transmission may have changed lately. Nosocomial GBS transmission in neonatal intensive care units (NICUs) is perhaps more common than expected. Two-year surveillance of all LOGBS cases in an NICU in the UK with serotyping and genome 
sequencing of GBS isolates together with neonatal rectal screening ${ }^{31}$ showed four clusters with 12 LOGBS cases, of which 11 were associated with at least one other LOGBS isolate. The authors concluded that even a single NICU case should be considered secondary to potential nosocomial transmission. LOGBS sepsis warrants prompt investigation and improved efforts for prevention of infection and prompt treatment because it may not necessarily indicate a sporadic occurrence. Hospital clusters of invasive GBS infections were investigated in a recent systematic review; crowding, inadequate disinfection of equipment and surfaces, unsatisfactory practices for prevention of infection, or a high patient-to-nurse ratio were implicated in the occurrence of LOGBS clusters. ${ }^{32}$

Usually, LOGBS presents a wider clinical spectrum than EOGBS and commonly manifests with bacteremia without an apparent focus of infection. Meningitis is significantly more frequent in LOGBS than in EOGBS $(31 \%$ vs $9 \%$ of cases) $)^{33,34}$ and may result in long-term neurodevelopmental sequelae of variable severity in up to $50 \%{ }^{35}$ and moderate-to-severe neurodevelopmental impairment in approximately $23 \%$ (95\% confidence interval [CI] 19 $26 \%$ ) of survivors. ${ }^{36}$ LOGBS rarely affects bones, joints, soft tissue, or the urinary tract (focal infections). ${ }^{1,33}$ Facial cellulitis and adenitis localized to the submandibular or parotid region are known to occur, and these infections are attributed to breastfeeding. ${ }^{37}$

In the absence of intervention, the gastrointestinal and/ or upper respiratory tracts of approximately 29-85\% (mean 50\%) of neonates born to GBS carrier mothers show colonization at birth. Most infants remain healthy, but $1-2 \%$ of the exposed neonates develop EOGBS. IAP prevents EOGBS and greatly reduces neonatal colonization at birth. ${ }^{38-42}$ Reportedly, GBS colonization of mucous surfaces occurs at the time of delivery in some infants who develop LOGBS. In a seminal study, Dillon reported that approximately $50 \%$ of infants with LOGBS showed colonization at birth with the same GBS serotype as the mother. ${ }^{14}$ However, whether this route of LOGBS transmission is common in real-world practice is unclear, particularly after the widespread use of IAP as a standard of care, which interrupts mother-to-infant transmission. IAP does not eradicate GBS carriage, and mothers remain a source of GBS transmission during the postpartum period. $^{25,28}$

IAP does not affect the LOGBS rates; however, it may delay the time of onset of LOGBS or reduce its severity. An Italian prospective cohort study that investigated 100 cases of LOGBS between 2003 and 2010 reported that infants who received IAP for any length of time were more likely to be older at the time of LOGBS presentation (median age at symptom onset 40.0 days, interquartile range [IQR] 25.0-62.0) than those who did not receive IAP (median age at symptom onset 24.0 days, IQR $15.0-$ 41.0 , odds ratio [OR] for early presentation $4.3,95 \% \mathrm{CI}$ $1.7-10.8, \mathrm{p}<0.01) .^{27}$ IAP exposure was significantly associated with mild rather than severe LOGBS $(p=0.03)$; however, the mechanisms underlying this association remain unclear. The severity of LOGBS is usually higher during the first few weeks of life; therefore, IAP may modify the routes of GBS transmission (from vertical to horizontal), with a consequent delay in disease onset and reduced disease severity. The unchanged rates of LOGBS following the widespread use of IAP support the role of postpartum colonization from maternal or non-maternal sources. $^{27}$

\section{Microbiological Aspects}

Ten immunologically distinct serotypes of GBS have been defined based on surface polysaccharides (Ia, Ib, II to IX). A systematic review and meta-analysis that included approximately 6500 strains obtained from neonatal GBS disease isolated worldwide between 2000 and 2017 observed that five serotypes (Ia, Ib, II, III, and V) account for $>97 \%$ of all cases of this disease. ${ }^{43}$ Serotype III strains are clinically the most important and account for approximately $50 \%$ of EOGBS and are the predominant strains in cases of LOGBS, with infection rates of $>70 \%{ }^{44-47}$ The predominance of serotype III is primarily attributable to the prevalence of a single clonal lineage defined as Clonal Complex 17 (CC17), transmitted worldwide. ${ }^{4-50}$ The monoclonal GBS nature of LOGBS etiology remains unexplained.

Maternal GBS colonization is the principal risk factor for LOGBS. Longitudinal studies during the postpartum period have shown that delayed GBS colonization occurs in infants born to GBS carrier mothers who receive IAP. ${ }^{51}$ Approximately $20 \%$ of neonates show colonization 1 month after birth with $>95 \%$ concordance between the maternal and infant GBS type. ${ }^{25,28,51}$ The distribution of GBS serotypes in colonized mother-neonate pairs resembles that of carriage observed during antenatal screening; serotype III-CC17 is detected but is not the exclusive serotype identified. $^{25}$

A recent study reported that compared with non-CC17, the $\mathrm{CC} 17$ strain is not associated with increased mother-to- 
child transmission during delivery, and no specific factor was correlated with $\mathrm{CC} 17$ colonization in infants, compared with other GBS types. Nevertheless, new GBS colonization that occurs in neonates between 21 and 60 days after birth is more frequently caused by $\mathrm{CC} 17$ as opposed to non-CC17 strains. $^{28}$

Meningeal tropism and propensity to cause meningitis (which is the most common clinical presentation of LOGBS) are attributable to the peculiar virulence traits of $\mathrm{CC} 17$ GBS. Compared with non-CC17 strains, $\mathrm{CC} 17$ GBS strains possess the exclusive surface adhesins, HvgA and Srr2, which promote increased binding to brain endothelial cells. While the molecular ligand of HvgA remains unidentified, a recent study has shown that Srr2 binds to integrins $\alpha 5 \beta 1$ and $\alpha v \beta 3$, which contribute to bacterial adhesion and invasion of brain endothelial cells and passage across the brain-blood barrier. Expression of these integrins is higher in neonatal than in adult brain vessels, which accounts for the greater tendency of $\mathrm{CC} 17$ to cause neurotropic disease in neonates with LOGBS. ${ }^{52}$

Comprehensive genomic analysis of $626 \mathrm{CC} 17$ isolates obtained worldwide between 1955 and 2016 to determine the underlying differences between carriage and diseaseassociated GBS strains showed that frequent mutations in the sensor protein of the regulatory transcriptional system CovS/R and in the serine/threonine-specific protein kinase were distinctive features of disease-specific $\mathrm{CC} 17$ isolates, which highlights the crucial role of fine-tuning of transcription and expression of virulence factors to modulate the pathogenicity of GBS. ${ }^{53}$ Parallel and convergent evolution (homoplastic events) were observed in other virulence components, such as in the capsule biosynthesis operon, the pilus, and surface protein Rib, which reflects the progressive temporal adaptation of CC17 GBS strains to host immune pressure. Among the alpha-like protein (Alp) members, a family of important adhesins detected in nearly all GBS strains, ${ }^{54}$ serotype III strains contain only the Rib variant, which reportedly is an important contributor to the virulence of the organism and to the development of protective immunity. Comparison of carriage-related isolates with disease-associated strains has sometimes shown a significant reduction in the number of tandem repeat units (which constitute the internal portion of Rib) in strains from neonatal infections, which modulate its exposure on the bacterial surface. ${ }^{55-57}$ The phase variation in the Alp proteins depends on their highly immunogenic properties, which are inversely correlated with the number of their repeated domains. ${ }^{55,56}$ Therefore, a smaller Rib enables serotype III CC-17 strains to easily escape the maternally transmitted antibodies and to be strongly selected in the course of disease. This confers a selective advantage in neonatal disease following maternal transmission. ${ }^{58}$

The success of $\mathrm{CC} 17$ is gaining much attention with recent reports on the emergence of a multidrug resistant (MDR) $\mathrm{CC} 17$ sub-clone. ${ }^{59-62}$ The GBS type III MDR $\mathrm{CC} 17$ sub-clone possesses integrative and conjugative elements (ICE) that confer additional genetic resistance to tetracycline, macrolides, and lincosamides and also highlevel resistance to streptomycin and kanamycin. ${ }^{59}$ Genomic studies have shown that the ICE elements replace the $16 \mathrm{~Kb}$ genome region that comprises the entire pathogenicity island encoding pilus $1 .^{59,60}$ Currently, two variants of ICE have been identified; these show a mosaic structure with modules derived from $S$. suis, S. dysgalactiae, S. pyogenes, and S. pneumoniae. ${ }^{59}$ The MDR CC17 sub-clone was firstly reported from the analysis of genomic GBS CC17 strains isolated from neonatal infections in Southern China in 2013-2014 (riferimento di Campisi). Nevertheless, retrospective analyses demonstrated that it has been circulating in Canada, ${ }^{60}$ as well as in Portugal ${ }^{61}$ and France ${ }^{62}$ since the year 2010.

Data from the surveillance network in France show a sustained increase in the incidence of LOGBS, with an overall $65 \%$ higher incidence over the past 20 years, along with an increase in prevalence of the hypervirulent $\mathrm{CC} 17$ GBS clone and its MDR sub-lineage in LOGBS. Specifically, the amikacin resistance rate among GBS strains from neonatal disease increased from $0 \%$ in 2007 to $18 \%$ in 2019, and the percentage of LOGBS infections caused by the MDR CC17 sub-clone was approximately $14 \%$ in $2019 .{ }^{62}$

Despite the availability of effective drugs, such as betalactams against GBS infections, the high plasticity of the mobile genetic elements that possess multiple antibiotic resistance genes in GBS type III CC17 MDR sub-clones is a significant concern for possible therapeutic failures, particularly in hospital settings such as in NICUs that are associated with extensive antibiotic use. Prolonged hospitalization predisposes neonates, particularly premature newborns to infection, with a high risk of horizontal GBS transmission and LOGBS.

\section{Risk Factors for Late-Onset Group B Streptococcus Disease}

RFs for LOGBS remain quite indeterminate; however, evidence from cohort and case-control studies indicates 
that ethnicity, prematurity, maternal GBS colonization, young maternal age, and HIV infection serve as RFs for LOGBS. Case reports or small case series have also implicated breast milk and twin delivery as RFs; however, published data do not support this view. We describe known and presumed RFs for LOGBS. Table 1 summarizes proven or suspected RFs and potential strategies (when available) to prevent LOGBS. However, maternal vaccination would prevent the largest number of LOGBS cases, according to vaccine efficacy and coverage in pregnant women. The hypothetical estimates of protection following maternal vaccination are reported in a recent systematic review. ${ }^{6}$ Unfortunately, no vaccination strategy will prevent LOGBS in very preterm neonates, since the transplacental transmission of maternal protective $\operatorname{IgG}$ antibodies mainly occurs after 34 weeks' gestation.

Table I Proven or Suspected Risk Factors for Group B Streptococcus Late-Onset Disease and Potential Strategies for Its Prevention

\begin{tabular}{|c|c|}
\hline Risk Factors & Potential Strategy for Preventing \\
\hline Ethnicity & NA \\
\hline Prematurity & $\begin{array}{l}\text { Prevention of maternal diseases that } \\
\text { can potentially lead to preterm birth } \\
\text { (obesity, hypertension, diabetes, heart } \\
\text { disease, others) } \\
\text { Prevention of inadequate lifestyles, } \\
\text { which increase the risk of preterm } \\
\text { birth } \\
\text { Prevention of healthcare associated } \\
\text { infections among in hospital premature } \\
\text { neonates }\end{array}$ \\
\hline $\begin{array}{l}\text { Maternal group } \\
\text { B streptococcus } \\
\text { colonization }\end{array}$ & $\begin{array}{l}\text { Maternal vaccination (possible } \\
\text { prevention of maternal group } \\
\text { B streptococcus colonization) } \\
\text { Prevention of mucous neonatal } \\
\text { colonization (hygiene measures, ie hand } \\
\text { washing) }\end{array}$ \\
\hline Young maternal age & NA \\
\hline $\begin{array}{l}\text { Exposure to the human } \\
\text { immunodeficiency virus }\end{array}$ & $\begin{array}{l}\text { Prevention of sexual transmission of } \\
\text { HIV in adults }\end{array}$ \\
\hline Breastfeeding & Prevention of maternal mastitis \\
\hline Twin delivery & $\begin{array}{l}\text { Prevention of twin deliveries, i.e.those } \\
\text { associated with assisted fertilization }\end{array}$ \\
\hline
\end{tabular}

Abbreviations: NA, not applicable; LOGBS, group B streptococcus late-onset disease.

\section{Ethnicity}

Cohort studies (mostly performed in the USA) have shown significant racial differences associated with LOGBS and that it affects a disproportionately high percentage of Black infants. GBS surveillance performed between 1999 and 2005 across 10 states that participated in the Active Bacterial Core surveillance/Emerging Infections Program Network in the USA reported that among 1036 patients with LOGBS, 28\% were Black and the overall incidence of LOGBS among the Black population was 2-fold higher than that among the White population (relative risk [RR] 2.0, 95\% CI 1.8-2.2). ${ }^{63}$ The correlation between race and prematurity, birth weight, maternal age, adequacy of prenatal care, or socioeconomic status could not be established, although socioeconomic status was not measured accurately. ${ }^{63}$ Schuchat et al ${ }^{64}$ retrospectively investigated 71 and 37 infants with invasive EOGBS and LOGBS, respectively, between 1982 and 1983 in Atlanta. Univariate analysis showed that $78 \%$ of LOGBS occurred in Black infants, independent of additional RFs. Multivariate analysis showed that Black ethnicity was strongly and independently associated with LOGBS (RR 35.1, CI 4.7-266.0). Nanduri et $\mathrm{al}^{20}$ investigated 1387 patients with LOGBS, based on active population-based and laboratory-based surveillance of invasive GBS disease (performed through Active Bacterial Core surveillance in the USA between 2006 and 2015); 588 infants (42.4\%) were Black, 706 (50.9\%) were White, and the remaining 94 (6.8\%) belonged to other races. Annual LOGBS incidence rates were higher in Black than in White infants (mean [range] rate ratio 2.9, CI 1.9-4.4 times). ${ }^{20}$ Lin et al ${ }^{65}$ investigated the RFs for LOGBS (disease onset or first positive culture between 7 and 180 days after birth) in the greater Houston area (1995-2000). These authors compared 122 infants with 122 controls matched for birth hospital and date of birth and observed that $50 \%$ of the infants were preterm, and $84 \%$ of these infants were born at $\geq 34$ weeks of gestation. The risk for LOGBS increased by 3.70 (95\% CI 1.35-10.1) in infants born to Black mothers. Although such disparities have reduced in recent decades, they are known to occur. In contrast, a reduced temporal trend in LOGBS was reported in Tennessee between 2009 and 2018. A study that investigated 359 infants with GBS disease (090 days of life) reported that 214 were diagnosed with LOGBS. The LOGBS rates among Black infants have shown a reduced temporal trend (RR $0.90,95 \%$ CI 0.84 
0.97, $\mathrm{p}=0.003$ ); however, no changes were observed among White infants (RR 0.99, 95\% CI 0.92-1.07, $\mathrm{p}=0.768$ ). ${ }^{66}$

\section{Prematurity}

LOGBS disproportionately affects premature neonates. ${ }^{2}$ Cohort and case-control studies have shown an increased risk of LOGBS in preterm infants, ${ }^{20,27,65}$ although a gradient showing an increased risk associated with decreasing gestational age is not clearly defined. The increased risk of LOGBS in preterm neonates is mainly attributable to their immature immune system, which cannot mount an adequate immune response against GBS; placental transfer of specific maternal IgG antibodies usually occurs during late pregnancy (serum levels of GBS type-specific antibodies increase to $75-80 \%$ of the maternal levels from 34 weeks of gestation, and levels before 34 weeks of gestation are only $20 \%$ of the maternal levels). ${ }^{67}$ Furthermore, survival of extremely preterm neonates has improved in recent times; an abnormal intestinal flora $^{68}$ associated with repeated antibiotic courses and prolonged hospital stay predisposes these neonates to nosocomial pathogen transmission. ${ }^{32,69,70}$

Jordan et $\mathrm{al}^{71}$ investigated 1726 patients with LOGBS in an active, population-based surveillance study performed across 10 states in the USA (between 1990 and 2005) and observed that although gestational age was known only in 455 of the 1726 patients, the rate of preterm neonates born before 37 weeks of gestation was high at $49 \%$. An Italian area-based study reported that preterm neonates accounted for approximately $33 \%$ of all cases of LOGBS, and an approximately 16-fold increase in incidence rates was observed in preterm neonates born before 34 weeks of gestation than in full-term neonates (incidence rates 3.8 and $0.24 / 1000$ live births, respectively). ${ }^{27} \mathrm{~A}$ recent study (between 2006 and 2015) by Nanduri et al reported from the Active Bacterial Core surveillance in the USA observed that approximately $40 \%$ of LOGBS cases occurred in infants born before 37 weeks' gestation, and the incidence of LOGBS was 6-fold higher in preterm than in full-term infants. ${ }^{20}$ Furthermore, mortality rates were disproportionately higher among preterm neonates (LOGBS case-fatality rates $3.4 \%$ and $7.8 \%$ among full-term and preterm infants, respectively). However, case-control studies may more accurately define the increased risk of LOGBS. Lin et a ${ }^{65}$ observed that each week of decreasing gestation was associated with an increased risk by a factor of 1.34. Similarly, Pintye et $\mathrm{al}^{72}$ reported a case-control study on RFs in 138 infants with LOGBS, which occurred before and after the implementation of universal screening and IAP. These authors investigated infants born in Washington State between 1992 and 2011, who were re-hospitalized between 7 and 89 days of life with a diagnosis of LOGBS (identified by the International Classification of Diseases- 9 codes) and observed that each week of decreasing gestation was associated with an increased risk of LOGBS by a factor of 1.24 .

\section{Maternal Group B Streptococcus Colonization}

Maternal GBS colonization is an important RF for LOGBS and is probably the main RF in full-term neonates, who usually have frequent and close contact with their mothers after discharge from the nursery. In contrast, preterm neonates admitted to hospitals are less frequently in close contact with their mothers and therefore they show a lower risk of maternal postpartum transmission of GBS; however, these neonates show a high risk of nosocomial infections, including GBS. ${ }^{73}$

In a prospective cohort study performed between 1977 and 1983, which included 50 infants with invasive GBS infection, 21 of 45 infants developed LOGBS. Maternal carriage with the same GBS serotype that caused LOGBS was detected at the time of delivery in $10(48 \%)$ of 21 mothers. ${ }^{14}$ Despite the small sample size of the study, maternal colonization rates were approximately 2-fold higher than those usually observed in pregnant women at VR sites. More recent studies have shown that prenatal colonization rates in mothers who deliver neonates with LOGBS are similar or moderately higher than those usually observed in pregnant women. An active, population-based surveillance study performed across 10 states in the USA (between 1990 and 2005) investigated 1726 infants with LOGBS and observed that only 182 women had documented results of prenatal GBS screening and 83 (46\%) showed positive results for GBS colonization. ${ }^{71}$ A French cohort study reported 438 GBS neonatal invasive infections (between January 2007 and December 2012), of which 264 were diagnosed as LOGBS. Among 102 mothers who underwent prenatal vaginal GBS screening, 32 (31\%) showed GBS colonization. Maternal VR colonization rates were comparable to those (27\%) observed in 169 infants with EOGBS during the same period. ${ }^{74}$ 
The two aforementioned case-control studies ${ }^{65,72}$ have accurately defined the risk of LOGBS in infants born to mothers with GBS colonization at VR sites. Pintye et al ${ }^{67}$ observed that 25 (33\%) of 77 mothers showed GBS colonization during prenatal screening, and the risk of LOGBS was approximately 2-fold higher among neonates who were born to mothers with GBS-positive prenatal screening (crude OR 2.06, CI 1.18-3.609). Lin et al reported that $38 \%$ (46 of 122) of mothers of neonates with LOGBS showed GBS colonization at VR sites during prenatal screening. The risk of LOGBS in infants of mothers who showed GBS-positive results on screening was increased (OR 4.15, 95\% CI 1.27-13.60). ${ }^{65}$ Multivariate analysis performed in a case-control study from South Africa that enrolled 46 infants showed that LOGBS was associated with maternal GBS colonization (OR 2.44, CI 0.88-6.79, $\mathrm{p}=0.088$ ) and GBS bacteriuria (OR 3.49, CI 1.17-10.40 $\mathrm{p}=0.025$ ), which serves as a marker for significant maternal colonization. However, approximately $40 \%$ of infants diagnosed with LOGBS in that study were exposed to HIV. $^{75}$

Maternal GBS carriage may vary over time; ${ }^{76}$ therefore, prenatal GBS colonization may not accurately predict maternal GBS status in the postpartum period. An Italian prospective area-based study that enrolled 100 infants with LOGBS between 2003 and $2010^{27}$ observed that 18 (27\%) of 67 mothers showed positive results on testing for GBS at VR sites during prenatal screening (prevalence of GBS carriage at VR sites in the same area was approximately $18 \%) .{ }^{77}$ However, rates of GBS positivity were higher in women in whom GBS carriage was evaluated at the time of LOGBS diagnosis. Approximately $66 \%$ of mothers showed positive results during prenatal screening and/or at the time the newborn was diagnosed with LOGBS. Many of these women had received IAP; therefore, postnatal transmission may be implicated in some cases. ${ }^{27}$

\section{Young Maternal Age}

Infants of teenage mothers ( $<20$ years) are predisposed to LOGBS. Multivariate analysis performed in a study by Schuchat et $\mathrm{al}^{64}$ indicated that maternal age $<20$ years was independently associated with LOGBS. Teenagers had an approximately 3 -fold higher risk of an infant with LOGBS (RR 2.96, CI 1.16-7.51). A case-control study reported an increased risk of LOGBS in neonates born to mothers aged $<20$ years (adjusted OR 2.32 [1.36-3.98], $\mathrm{p}=0.002){ }^{72}$ Although young maternal age was more strongly associated with LOGBS among infants born before the implementation of universal GBS screening, this difference was not statistically significant. ${ }^{11,20,63,72,78}$ The underlying mechanism that contributes to the association between young maternal age and LOGBS is unknown; however, young mothers tend to have higher GBS carriage at VR sites and also a weaker immune response or a higher incidence of co-infections, which favor GBS transmission. ${ }^{11,20,63,78}$

\section{Exposure to the Human Immunodeficiency Virus}

A few studies suggest a higher risk of LOGBS in newborns with HIV exposure or infection, despite the similar prevalence of colonization observed in such cases. A cohort study from Belgium reported seven episodes of neonatal GBS infections (LOGBS, $n=6$ ) among HIVexposed uninfected neonates. The incidence of GBS infection was significantly higher in infants born to HIVuninfected mothers. ${ }^{79}$ A hospital-based surveillance study performed in Soweto (South Africa, 2004-2008) showed a higher incidence of invasive GBS disease in HIVexposed than HIV-unexposed infants (4.46 cases/1000 live births [95\% CI 3.85-5.13] vs 1.98 cases/1000 live births [95\% CI 1.71-2.28]). The higher incidence was most evident in infants with LOGBS (2.36 vs 0.74 cases/ 1000 live births, RR 3.18, 95\% CI 2.34-4.36). ${ }^{80} \mathrm{~A}$ casecontrol study in South Africa (2012-2014) of 122 neonates with invasive GBS disease (of which 56 were diagnosed with LOGBS) reported that the incidence of EOGBS was similar between HIV-exposed and unexposed neonates (1.1 vs $1.5 \%, \mathrm{p}=0.5$ ). However, the risk of LOGBS was 4.7-fold higher in HIV-exposed than in HIV-unexposed infants $(2.3$ vs $0.5 \%, p<0.001)$. Although not explicitly stated, the study appears to have been prospectively designed. $^{75}$

\section{Breastfeeding}

The literature that describes breast milk-associated cases of LOGBS is based nearly exclusively on findings of case reports or small case series. ${ }^{24,37,81-86}$ Some investigators are of the opinion that breastfeeding may contribute to recurrent episodes of GBS transmission to the newborn, ${ }^{24}$ which promotes gut translocation and GBS invasion of the bloodstream. Breast milk with GBS contamination is sometimes associated with symptomatic maternal mastitis, ${ }^{24}$ but more often with silent mastitis, ${ }^{25}$ with GBS counts sometimes high. ${ }^{24-27}$ However, some study reports cases of 
LOGBS with GBS confirmed by polymerase chain reaction assays on breast milk despite a sterile culture. ${ }^{83}$ Often GBS contaminated breast milk leads to heavy neonatal colonization, ${ }^{25}$ and oral administration of GBS is shown to cause systemic infection in animal studies. ${ }^{22}$ A recent comprehensive review that summarizes data from 48 cases has shown that breast milk-associated LOGBS has a high recurrence rate and that LOGBS may occur even without RFs other than contaminated breast milk; however, ${ }^{24}$ the mechanism underlying breast milk contamination with GBS is unclear. The "retrograde theory" assumes that neonatal colonization occurs at birth and colonization of the oral mucosa contaminates the maternal mammary ducts, owing to the negative pressure created during the sucking action of the newborn. Multiplication of GBS within the mammary ducts increases the bacterial load in milk and reexposes the neonate to infection during breastfeeding, which results in persistent exposure/colonization of both the neonate and the mother. ${ }^{37,82,84,86}$ Alternatively, GBS might be transmitted to the mammary glands following translocation from the gastrointestinal tract through the lymphatics. ${ }^{87}$ This additional mode of transmission may explain GBS in the breast milk in women who have never breastfed their newborn but show positive results for VR site colonization. ${ }^{70}$

However, the only case-control study published to date failed to retrospectively confirm an association between breastfeeding and LOGBS. ${ }^{72}$ Approximately $0.8-3.5 \%$ of women contain GBS in breast milk; $;^{88,89}$ most breastfed infants do not develop LOGBS. Breast milk has immunemodulating and antimicrobial components (natural secretory immunoglobulin (sIg)A and GBS-specific $\operatorname{IgG})^{26,90}$ and plays a key role in protection of infants against infections. A deficiency of these protective agents in breast milk, rather than breastfeeding per se, may increase the risk of LOGBS. ${ }^{26,91}$ Le Doare et $\mathrm{al}^{26}$ prospectively investigated 750 Gambian mother-infant pairs who were followed-up until day 89 of life and observed that $\operatorname{sIgA}$ capsular antibodies in colostrum and key immunomodulatory cytokines (tumor necrosis factor- $\alpha$, interleukin [IL]- 6 , IL-10, and transforming growth factor- $\beta$ ) were associated with infant serotype III and V GBS colonization, acquisition, and clearance up to 3 months of life. A recent South African study by Dangor et al ${ }^{91}$ which included 31 cases of LOGBS reported that breast milk sIgA serotype III antibody concentrations $\geq 2.52 \mu \mathrm{g} / \mathrm{mL}$ reduced the risk of LOGBS in infants by $90 \%$.

\section{Twins}

Limited reports in the available literature describe the association between LOGBS and twins. Most studies are case reports or case series, and definitive conclusions cannot be drawn from their findings. Twin pregnancies are associated with higher preterm delivery rates, and prematurity is an important RF for LOGBS. Therefore, it is reasonable to conclude that theoretically, twin pregnancies may be associated with an increased risk of LOGBS. Moreover, twins are exposed to the same maternal genital bacteria, breast milk, and shared nursing care and hospital environments and share genetic susceptibility to infections. A retrospective cohort study performed by Bizzarro et al $^{92}$ compared the sepsis concordance rates between 170 monozygotic and 665 dizygotic twin pairs, and the authors observed a significant genetic susceptibility to late-onset sepsis from all pathogens (independent of additional known RFs, such as gestational age or birth weight). In fact, $49.0 \%(\mathrm{p}=0.002)$ of the variance in susceptibility to late-onset sepsis was attributable exclusively to genetic factors and $51.0 \%(\mathrm{p}=0.001)$ to residual environmental factors.

Edwards et al investigated six sets of twins in whom at least one infant had LOGBS disease. ${ }^{93}$ A recent review that investigated the association between twin pregnancies and the risk of LOGBS summarizes 13 articles published between 1976 and 2017. ${ }^{90}$ The authors observed that among 17 siblings or triplets, most (9 of 17) were born preterm and all but one infant showed serotype III GBS infection on GBS serotyping. LOGBS showed variable delay in presentation in each twin (between 0 hours and 52 days). ${ }^{93,94}$ However, these articles did not discuss comparisons with singleton pregnancies.

\section{Conclusion}

Significant gaps exist in the knowledge of LOGBS; limited studies have discussed the RFs for LOGBS, although this information would be useful to implement prevention strategies against LOGBS. Most reports in the literature are retrospectively designed case-control studies, which precludes accurate data collection, and it is not possible to conclusively establish the contributory role of variables such as positive breast milk contamination and maternal GBS colonization at the time of diagnosis of LOGBS, owing to the retrospective design. Well-designed casecontrol studies are warranted to accurately determine the role of RFs. The best design for such research would be 
a large scale, prospective nested case-control study with detailed information available at the time of LOGBS diagnosis in patients and age-matched controls. The knowledge of risk factors and mechanisms underlying LOGBS will contribute to its prevention. Maternal vaccination is likely to be the best preventive strategy of the future.

\section{Acknowledgments}

We are grateful to dr. Monica Imperi (Istituto Superiore di Sanità) who revised the initial draft of the manuscript and gave very valuable suggestions.

\section{Funding}

This research received no external funding.

\section{Disclosure}

The authors declare no conflict of interest.

\section{References}

1. Edwards MS, Nizet V, Baker CJ. Group B streptococcal infections. In: Remington and Klein's Infectious Diseases of the Fetus and Newborn Infant. 8th ed. Elsevier; 2016:411-456.

2. Dhudasia MB, Flannery DD, Pfeifer MR, Puopolo KM. Updated guidance: prevention and management of perinatal group B Streptococcus infection. Neoreviews. 2021;22(3):e177-e188. doi:10.1542/neo.22-3-e177

3. Verani JR, McGee L, Schrag SJ; Division of Bacterial Diseases, National Center for Immunization and Respiratory Diseases, Centers for Disease Control and Prevention (CDC). Prevention of perinatal group B Streptococcal disease-revised guidelines from CDC, 2010. MMWR Recomm Rep. 2010;59(RR-10):1-36.

4. Di Renzo GC, Melin P, Berardi A, et al. Intrapartum GBS screening and antibiotic prophylaxis: a European consensus conference. J Matern Fetal Neonatal Med. 2015;28(7):766-782. doi:10.3109/ 14767058.2014 .934804

5. Edmond KM, Kortsalioudaki C, Scott S, et al. Group B streptococcal disease in infants aged younger than 3 months: systematic review and meta-analysis. Lancet. 2012;379(9815):547-556. doi:10.1016/S01406736(11)61651-6

6. Seale AC, Bianchi-Jassir F, Russell NJ, et al. Estimates of the burden of group B Streptococcal disease worldwide for pregnant women, stillbirths, and children. Clin Infect Dis. 2017;65(suppl_2):S200S219. doi:10.1093/cid/cix664

7. Liu L, Oza S, Hogan D, et al. Global, regional, and national causes of under-5 mortality in 2000-15: an updated systematic analysis with implications for the sustainable development goals. Lancet. 2016;388 (10063):3027-3035. doi:10.1016/S0140-6736(16)31593-8

8. Baker CJ, Barrett FF. Transmission of group B streptococci among parturient women and their neonates. J Pediatr. 1973;83(6):919-925. doi:10.1016/S0022-3476(73)80524-4

9. Gardner SE, Yow MD, Leeds LJ, Thompson PK, Mason EO, Clark DJ. Failure of penicillin to eradicate group B Streptococcal colonization in the pregnant woman. A couple study. Am J Obstet Gynecol. 1979;135(8):1062-1065. doi:10.1016/0002-9378(79) 90737-3

10. Gardner SE, Mason EO, Yow MD. Community acquisition of group B Streptococcus by infants of colonized mothers. Pediatrics. 1980;66 (6):873-875
11. Anthony BF, Okada DM, Hobel CJ. Epidemiology of group B Streptococcus: longitudinal observations during pregnancy. J Infect Dis. 1978;137(5):524-530. doi:10.1093/infdis/137.5.524

12. Pass MA, Gray BM, Khare S, Dillon HC. Prospective studies of group B streptococcal infections in infants. J Pediatr. 1979;95 (3):437-443. doi:10.1016/S0022-3476(79)80531-4

13. Easmon CS, Hastings MJ, Blowers A, et al. Epidemiology of group B streptococci: one year's experience in an obstetric and special care baby unit. Br J Obstet Gynaecol. 1983;90(3):241-246. doi:10.1111/ j.1471-0528.1983.tb08617.x

14. Dillon HC, Khare S, Gray BM. Group B streptococcal carriage and disease: a 6-year prospective study. J Pediatr. 1987;110(1):31-36. doi:10.1016/S0022-3476(87)80283-4

15. Boyer KM, Gotoff SP. Prevention of early-onset neonatal group B streptococcal disease with selective intrapartum chemoprophylaxis. $N$ Engl $J$ Med. 1986;314(26):1665-1669. doi:10.1056/NEJM198606263142603

16. Tuppurainen N, Hallman M. Prevention of neonatal group B streptococcal disease: intrapartum detection and chemoprophylaxis of heavily colonized parturients. Obstet Gynecol. 1989;73 (4):583-587.

17. Garland SM, Fliegner JR. Group B streptococcus (GBS) and neonatal infections: the case for intrapartum chemoprophylaxis. Aust N Z J Obstet Gynaecol. 1991;31(2):119-122. doi:10.1111/j.1479828X.1991.tb01797.x

18. Cantey JB, Baldridge C, Jamison R, Shanley LA. Late and very late onset group B Streptococcus sepsis: one and the same? World J Pediatr. 2014;10(1):24-28. doi:10.1007/s12519-014-0450-8

19. Benitz WE, Gould JB, Druzin ML. Risk factors for early-onset group B streptococcal sepsis: estimation of odds ratios by critical literature review. Pediatrics. 1999;103(6):e77. doi:10.1542/peds.103.6.e77

20. Nanduri SA, Petit S, Smelser C, et al. Epidemiology of invasive early-onset and late-onset group B Streptococcal disease in the United States, 2006 to 2015: multistate laboratory and population-based surveillance. JAMA Pediatr. 2019;173(3):224-233. doi:10.1001/jamapediatrics.2018.4826

21. Weindling AM, Hawkins JM, Coombes MA, Stringer J. Colonisation of babies and their families by group B streptococci. Br Med J (Clin Res Ed). 1981;283(6305):1503-1505. doi:10.1136/ bmj.283.6305.1503

22. Weisman LE, McKinney L, Villalobos R. Systemic group B streptococcal disease in the neonate: characterization of an oral colonization model using the suckling rat. Microbiol Immunol. 1990;34(9):755-764. doi:10.1111/j.1348-0421.1990.tb01053.x

23. Vaz MJ, Purrier SA, Bonakdar M, Chamby AB, Ratner AJ, Randis TM. The impact of circulating antibody on group B Streptococcus intestinal colonization and invasive disease. Infect Immun. 2020;89(1):e00348-20. doi:10.1128/IAI.00348-20

24. Filleron A, Lombard F, Jacquot A, et al. Group B streptococci in milk and late neonatal infections: an analysis of cases in the literature. Arch Dis Child Fetal Neonatal Ed. 2014;99(1):F41-7. doi:10.1136/ archdischild-2013-304362

25. Berardi A, Rossi C, Creti R, et al. Group B streptococcal colonization in 160 mother-baby pairs: a prospective cohort study. $J$ Pediatr. 2013;163(4):1099-104.e1. doi:10.1016/j.jpeds.2013.05.064

26. Le Doare K, Bellis K, Faal A, et al. SIgA, TGF- $\beta 1$, IL-10, and TNF $\alpha$ in colostrum are associated with infant group B Streptococcus colonization. Front Immunol. 2017;8:1269. doi:10.3389/ fimmu.2017.01269

27. Berardi A, Rossi C, Lugli L, et al. Group B streptococcus late-onset disease: 2003-2010. Pediatrics. 2013;131(2):e361-8. doi:10.1542/ peds.2012-1231

28. Tazi A, Plainvert C, Anselem O, et al. Risk factors for infant colonization by hypervirulent $\mathrm{CC} 17$ group B Streptococcus: toward the understanding of late-onset disease. Clin Infect Dis. 2019;69 (10):1740-1748. doi:10.1093/cid/ciz033 
29. Steere AC, Aber RC, Warford LR, et al. Possible nosocomial transmission of group B streptococci in a newborn nursery. $J$ Pediatr. 1975;87(5):784-787. doi:10.1016/S0022-3476(75) 80311-8

30. Paredes A, Wong P, Mason EO, Taber LH, Barrett FF. Nosocomial transmission of group B Streptococci in a newborn nursery. Pediatrics. 1977;59(5):679-682.

31. Jauneikaite E, Kapatai G, Davies F, et al. Serial Clustering of LateOnset Group B Streptococcal Infections in the Neonatal Unit: A Genomic Re-evaluation of Causality. Clin Infect Dis. 2018Aug 31;67(6):854-860. doi:10.1093/cid/ciy174

32. Collin SM, Lamb P, Jauneikaite E, et al. Hospital clusters of invasive group B Streptococcal disease: a systematic review. J Infect. 2019;79 (6):521-527. doi:10.1016/j.jinf.2019.11.008

33. Puopolo KM, Lynfield R, Cummings JJ. Management of infants at risk for group B Streptococcal disease. Pediatrics. 2019;144(2): e20191881. doi:10.1542/peds.2019-1881

34. Berardi A, Lugli L, Rossi C, et al. Infezioni da Streptococco B Della Regione Emilia Romagna. Neonatal bacterial meningitis. Minerva Pediatr. 2010;62(3 Suppl 1):51-54.

35. Libster R, Edwards KM, Levent F, et al. Long-term outcomes of group B streptococcal meningitis. Pediatrics. 2012;130(1):e8-e15. doi:10.1542/peds.2011-3453

36. Kohli-Lynch M, Russell NJ, Seale AC, et al. Neurodevelopmental impairment in children after group B Streptococcal disease worldwide: systematic review and meta-analyses. Clin Infect Dis. 2017;65 (suppl_2):S190-S199. doi:10.1093/cid/cix663

37. Bertini G, Dani C. Group B streptococcal late-onset sepsis with submandibular phlegmon in a premature infant after beginning of breast-feeding. J Matern Fetal Neonatal Med. 2008;21(3):213-215. doi:10.1080/14767050801924886

38. Berardi A, Cattelani C, Creti R, et al. Group B streptococcal infections in the newborn infant and the potential value of maternal vaccination. Expert Rev Anti Infect Ther. 2015;13(11):1387-1399. doi:10.1586/14787210.2015.1079126

39. De Cueto M, Sanchez MJ, Sampedro A, et al. Timing of intrapartum ampicillin and prevention of vertical transmission of group B streptococcus. Obstet Gynecol. 1998;91:112-114. doi:10.1016/ S0029-7844(97)00587-5

40. Boyer KM, Gadzala CA, Kelly PD, et al. Selective intrapartum chemoprophylaxis of neonatal group B streptococcal early-onset disease. III. Interruption of mother-to-infant transmission. J Infect Dis. 1983;148:810-816. doi:10.1093/infdis/148.5.810

41. Berardi A, Rossi C, Biasini A, et al. Efficacy of intrapartum chemoprophylaxis less than 4 hours duration. J Matern Fetal Neonatal Med. 2011;24(4):619-625. doi:10.3109/14767058.2010.511347

42. Berardi A, Rossi C, Guidotti I, et al. Factors associated with intrapartum transmission of group B Streptococcus. Pediatr Infect Dis J. 2014;33:1211-1215. doi:10.1097/INF.0000000000000439

43. Madrid L, Seale AC, Kohli-Lynch M, et al. Infant group B Streptococcal disease incidence and serotypes worldwide: systematic review and meta-analyses. Clin Infect Dis. 2017;65(suppl_2): S160-S172. doi:10.1093/cid/cix656.

44. Melin P, Efstratiou A. Group B streptococcal epidemiology and vaccine needs in developed countries. Vaccine. 2013;31(Suppl 4): D31-D42. doi:10.1016/j.vaccine.2013.05.012

45. Creti R, Imperi M, Berardi A, et al.; Italian Neonatal GBS Infections Working Group. Neonatal group B Streptococcus infections: prevention strategies, clinical and microbiologic characteristics in 7 years of surveillance. Pediatr Infect Dis J. 2017;36(3):256-262. doi:10.1097/ INF.0000000000001414

46. O'Sullivan CP, Lamagni T, Patel D, et al. Group B streptococcal disease in UK and Irish infants younger than 90 days, 2014-15: a prospective surveillance study. Lancet Infect Dis. 2019;19 (1):83-90. doi:10.1016/S1473-3099(18)30555-3
47. Lohrmann F, Berg A, Wicker E, et al. Prevalence of capsular serotype, pilus island distribution, and antibiotic resistance in pediatric and adult invasive group B Streptococcus isolates: data from a nationwide prospective surveillance study in germany. Pediatr Infect Dis J. 2021;40(1):76-82. doi:10.1097/ INF.0000000000002943

48. Jamrozy D, Bijlsma MW, de Goffau MC, et al. Increasing incidence of group B streptococcus neonatal infections in the Netherlands is associated with clonal expansion of $\mathrm{CC} 17$ and CC23. Sci Rep. 2020;10(1):9539. doi:10.1038/s41598-020-66214-3

49. Ding Y, Wang Y, Hsia Y, Russell N, Heath PT. Systematic review and meta-analyses of incidence for group B Streptococcus disease in infants and antimicrobial resistance, China. Emerg Infect Dis. 2020;26(11):2651-2659. doi:10.3201/ eid2611.181414

50. Kekic D, Gajic I, Opavski N, et al. Trends in molecular characteristics and antimicrobial resistance of group B streptococci: a multicenter study in Serbia, 2015-2020. Sci Rep. 2021;11(1):540. doi:10.1038/s41598-020-79354-3

51. Toyofuku M, Morozumi M, Hida M, et al. Effects of intrapartum antibiotic prophylaxis on neonatal acquisition of group B Streptococci. $J$ Pediatr. 2017;190:169-173.e1. doi:10.1016/j. jpeds.2017.07.039

52. Deshayes de Cambronne R, Fouet A, Picart A, et al. CC17 group B Streptococcus exploits integrins for neonatal meningitis development. J Clin Invest. 2021;131(5):e136737. doi:10.1172/ JCI136737

53. Almeida A, Rosinski-Chupin I, Plainvert C, et al. Parallel evolution of group B Streptococcus hypervirulent clonal complex 17 unveils new pathoadaptive mutations. mSystems. 2017;2(5):e00074-17. doi: 10.1128/mSystems.00074-17

54. Lachenauer CS, Creti R, Michel JL, Madoff LC. Mosaicism in the alpha-like protein genes of group B streptococci. Proc Natl Acad Sci USA. 2000;97(17):9630-9635. doi:10.1073/pnas.97.17.9630

55. Gravekamp C, Rosner B, Madoff LC, Fischetti VA. Deletion of repeats in the alpha $\mathrm{C}$ protein enhances the pathogenicity of group B streptococci in immune mice. Infect Immun. 1998;66 (9):4347-4354. doi:10.1128/IAI.66.9.4347-4354.1998

56. Madoff LC, Michel JL, Gong EW, Kling DE, Kasper DL. Group B streptococci escape host immunity by deletion of tandem repeat elements of the alpha C protein. Proc Natl Acad Sci USA. 1996;93:4131-4136. doi:10.1073/pnas.93.9.4131

57. Whelan F, Lafita A, Griffiths SC, et al. Defining the remarkable structural malleability of a bacterial surface protein Rib domain implicated in infection. Proc Natl Acad Sci USA. 2019;116 (52):26540-26548. doi:10.1073/pnas.1911776116

58. Almeida A, Villain A, Joubrel C, et al. Whole-genome comparison uncovers genomic mutations between group B streptococci sampled from infected newborns and their mothers. $J$ Bacteriol. 2015;197:3354-3366. doi:10.1128/JB.00429-15

59. Campisi E, Rosini R, Ji W, et al. Genomic analysis reveals multi-drug resistance clusters in group B Streptococcus CC17 hypervirulent isolates causing neonatal invasive disease in Southern Mainland China. Front Microbiol. 2016;7:1265. doi:10.3389/fmicb.2016.01265

60. Teatero S, Ramoutar E, McGeer A, et al. Clonal complex 17 group B Streptococcus strains causing invasive disease in neonates and adults originate from the same genetic pool. Sci Rep. 2016;6:20047. doi:10.1038/srep20047

61. Martins ER, Pedroso-Roussado C, Melo-Cristino J, Ramirez M; Portuguese Group for the Study of Streptococcal Infections. Streptococcus agalactiae causing neonatal infections in Portugal (2005-2015): diversification and emergence of a CC17/PI-2b multidrug resistant sublineage. Front Microbiol. 2017;8:499. doi:10.3389/ fmicb.2017.00499 
62. Plainvert C, Hays C, Touak G, et al. Multidrug-resistant hypervirulent group B Streptococcus in neonatal invasive infections, France, 2007-2019. Emerg Infect Dis. 2020;26(11):2721-2724. doi:10.3201/ eid2611.201669

63. Phares CR, Lynfield R, Farley MM, et al. Epidemiology of invasive group B streptococcal disease in the United States, 1999-2005. JAMA. 2008;299(17):2056-2065. doi:10.1001/jama.299.17.2056

64. Schuchat A, Oxtoby M, Cochi S, et al. Population-based risk factors for neonatal group B streptococcal disease: results of a cohort study in metropolitan Atlanta. $J$ Infect Dis. 1990;162:672-677. doi:10.1093/infdis/162.3.672

65. Lin FY, Weisman LE, Troendle J, Adams K. Prematurity is the major risk factor for late-onset group B streptococcus disease. J Infect Dis. 2003;188(2):267-271. doi:10.1086/376457

66. Hamdan L, Vandekar S, Spieker AJ, et al. Epidemiological trends of racial differences in early- and late-onset group B Streptococcus disease in Tennessee. Clin Infect Dis. 2020; ciaa1511. doi:10.1093/ $\mathrm{cid} / \mathrm{ciaa} 1511$

67. Lin FY, Philips JB, Azimi PH, et al. Level of maternal antibody required to protect neonates against early-onset disease caused by type 1a group B streptococcus: a multicenter, seroepidemiology study. J Infect Dis. 2001;184:1022-1028. doi:10.1086/323350

68. Ficara M, Pietrella E, Spada C. Changes of intestinal microbiota in early life. J Matern Fetal Neonatal Med. 2020;33:1036-1043. doi:10.1080/14767058.2018.1506760

69. Barbadoro P, Marigliano A, Savini S, D’Errico MM, Prospero E. Group B Streptococcal sepsis: an old or ongoing threat? Am J Infect Control. 2011;39(8):e45-e48. doi:10.1016/j.ajic.2010.12.017

70. Berardi A, Guidotti I, Creti R, et al. Two overlapping clusters of group B Streptococcus late-onset disease in a neonatal intensive care unit. Pediatr Infect Dis J. 2018;37(11):1160-1164. doi:10.1097/ INF.0000000000001987

71. Jordan HT, Farley MM, Craig A, et al. Revisiting the need for vaccine prevention of late-onset neonatal group B streptococcal disease: a multistate, population-based analysis. Pediatr Infect Dis J. 2008;27(12):1057-1064. doi:10.1097/INF.0b013e318180b3b9.

72. Pintye J, Saltzman B, Wolf E, Crowell CS. Risk factors for late-onset group B Streptococcal disease before and after implementation of universal screening and intrapartum antibiotic prophylaxis. $J$ Pediatric Infect Dis Soc. 2016;5(4):431-438. doi:10.1093/jpids/piv067

73. MacFarquhar JK, Jones TF, Woron AM, et al. Outbreak of late-onset group B Streptococcus in a neonatal intensive care unit. Am J Infect Control. 2010;38:283-288. doi:10.1016/j.ajic.2009.08.011

74. Joubrel C, Tazi A, Six A, et al. Group B streptococcus neonatal invasive infections, France 2007-2012. Clin Microbiol Infect. 2015;21:910-916. doi:10.1016/j.cmi.2015.05.039

75. Dangor Z, Lala SG, Cutland CL, et al. Burden of invasive group B Streptococcus disease and early neurological sequelae in South African infants. PLoS One. 2015;10:e0123014. doi:10.1371/journal. pone. 0123014

76. Hansen SM, Uldbjerg N, Killian M, Sorensen UB. Dynamics of Streptococcus agalactiae colonization in women during and after pregnancy and in their infants. J Clin Microbiol. 2004;42:83-89. doi:10.1128/JCM.42.1.83-89.2004

77. Berardi A, Di Fazzio G, Gavioli S, et al. Universal antenatal screening for group B streptococcus in Emilia-Romagna. J Med Screen. 2011;18(2):60-64. doi:10.1258/jms.2011.011023

78. Fraser AM, Brockert JE, Ward RH. Association of young maternal age with adverse reproductive outcomes. $N$ Engl J Med. 1995;332:1113-1117. doi:10.1056/NEJM199504273321701
79. Epalza C, Goetghebuer T, Hainaut M, et al. High incidence of invasive group B streptococcal infections in HIV-exposed uninfected infants. Pediatrics. 2010;126(3):e631-e638. doi:10.1542/peds.20100183

80. Cutland CL, Schrag SJ, Thigpen MC, et al. Increased risk for group B Streptococcus sepsis in young infants exposed to HIV, Soweto, South Africa, 2004-2008. Emerg Infect Dis. 2015;21:638-645. doi:10.3201/eid2104.141562

81. Zimmermann P, Gwee A, Curtis N. The controversial role of breast milk in GBS late-onset disease. J Infect. 2017;74(Suppl 1):S34-S40. doi:10.1016/S0163-4453(17)30189-5

82. Kenny JF, Zedd AJ. Recurrent group B streptococcal disease in an infant associated with the ingestion of infected mother's milk J Pediatr. 1977;91:158-159. doi:10.1016/S0022-3476(77)80473-3

83. Lanari M, Serra L, Cavrini F, Liguori G, Sambri V. Late-onset group B streptococcal disease by infected mother's milk detected by polymerase chain reaction. New Microbiol. 2007;30:253-254.

84. Byrne PA, Miller C, Justus K. Neonatal group B streptococcal infection related to breast milk. Breastfeed Med. 2006;1:263-270. doi:10.1089/bfm.2006.1.263

85. Bingen E, Denamur E, Lambert-Zechovsky N, et al. Analysis of DNA restriction fragment length polymorphism extends the evidence for breast milk transmission in Streptococcus agalactiae late-onset neonatal infection. J Infect Dis. 1992;165:569-573. doi:10.1093/ infdis/165.3.569

86. Dinger J, Muller D, Pargac N, Schwarze R. Breast milk transmission of group B streptococcal infection. Pediatr Infect Dis J. 2002;21:567-568. doi:10.1097/00006454-200206000-00017

87. Perez PF, Doré J, Leclerc M, et al. Bacterial imprinting of the neonatal immune system: lessons from maternal cells? Pediatrics. 2007;119(3):e724-e732. doi:10.1542/peds.2006-1649

88. Kubın V, Mrastikova H, Paulova M, Motlova J, Frane`k J. Group B Streptococci in the milk of lactating mothers. Zentralbl Bakteriol Mikrobiol Hyg A. 1987;265:210-217.

89. Burianova I, Paulova M, Cermak P, Janota J. Group B streptococcus colonization of breast milk of group B streptococcus positive mothers. J Hum Lact. 2013;29:586-590. doi:10.1177/ 0890334413479448

90. Feildel-Fournial C, Launay E, Caillon J, Thomas E, Boscher C, Grasle Guen C. What is the optimal management for the asymptomatic twin after diagnosis of late-onset group B streptococcal disease? Arch Dis Child. 2019;104:401-405. doi:10.1136/archdischild-2018316170

91. Dangor Z, Khan M, Kwatra G, et al. The association between breastmilk group B Streptococcal capsular antibody levels and late-onset disease in young infant. Clin Infect Dis. 2020;70:1110-1114.

92. Bizzarro MJ, Jiang Y, Hussain N, Gruen JR, Bhandari V, Zhang H. The impact of environmental and genetic factors on neonatal late-onset sepsis. J Pediatr. 2011;158:234-238.e1. doi:10.1016/j. jpeds.2010.07.060

93. Edwards MS, Jackson CV, Baker CJ. Increased risk of group B streptococcal disease in twins. JAMA. 1981;245:2044-2046. doi:10.1001/jama.1981.03310450036019

94. Matsubara K, Hoshina K, Kondo M, et al. Group B streptococcal disease in infants in the first year of life: a nationwide surveillance study in Japan, 2011-2015. Infection. 2017;45:449-458. doi:10.1007/ s15010-017-0995-2 


\section{Publish your work in this journal}

Infection and Drug Resistance is an international, peer-reviewed openaccess journal that focuses on the optimal treatment of infection (bacterial, fungal and viral) and the development and institution of preventive strategies to minimize the development and spread of resistance. The journal is specifically concerned with the epidemiology of antibiotic resistance and the mechanisms of resistance development and diffusion in both hospitals and the community. The manuscript management system is completely online and includes a very quick and fair peerreview system, which is all easy to use. Visit http://www.dovepress.com testimonials.php to read real quotes from published authors. 